\title{
Laboratory characterization of directional dependence of permeability for porous asphalt mixtures 1
}

\author{
Jun Chen, Ph.D. \\ Associate Professor \\ College of Civil and Transportation Engineering \\ Hohai University \\ 1 Xikang Road, Nanjing PRC, 210098 \\ Phone: +86-13813982728 \\ Email: jun.chen@hhu.edu.cn \\ Tao Tang, Ph.D. Candidate \\ School of Transportation \\ Southeast University \\ 2 SiPaiLou, Nanjing PRC, 210096 \\ Phone: +86-13675179133 \\ Email:230149519@seu.edu.cn \\ Yuqing Zhang*, Ph.D. \\ Lecturer \\ Aston Institute of Materials Research \\ School of Engineering and Applied Science \\ Aston University \\ Aston Triangle, Birmingham, B4 7ET, U.K. \\ Phone: +44 (0) 121-204-3391 \\ Email: y.zhang10@aston.ac.uk

\section{${ }^{*}$ Corresponding Author}

${ }^{1}$ This is an Accepted Manuscript of an article published by Springer in Materials and Structures. The final publication is available online via https://doi.org/10.1617/s11527-017-1081-z 


\begin{abstract}
Water permeability is an important property for porous asphalt mixtures. Previous numerical modeling showed that the permeability of the porous asphalt mixtures varies in different directions and a single permeability cannot accurately evaluate the mixture's directional permeability. To investigate the direction-dependent water permeability of the porous asphalt mixtures, a unidirectional permeameter was used to measure the permeability in twelve directions in the vertical plane (parallel to compaction direction) and twelve directions in the horizontal plane (perpendicular to the compaction direction) on two open graded friction course (OGFC) mixtures with different nominal maximum aggregate sizes, i.e., OGFC-13 and OGFC-10. Furthermore, a new multidirectional permeameter was designed which can control the rainfall intensity and adjust transverse slope to simulate the actual water flow process in pavement. The multidirectional permeability and void saturation of eight porous asphalt mixtures were determined by the multidirectional permeameter. Results show that the porous asphalt mixtures demonstrate direction-dependent permeability properties in both vertical and horizontal planes, whereas the dependence is less in the horizontal plane than that in the vertical plane. In the vertical plane, the minimum permeability occurs in the vertical direction and the maximum value occurs in the horizontal direction. In the horizontal plane, the permeability differs in different directions, but has no obvious relationship with directions. Increasing the air void content and the nominal maximum aggregate size of the mixtures can reduce the directional difference of the permeability. The void inside porous mixture cannot be entirely occupied by water when surface runoff occurs. Increasing the air void content and aggregate particle size can lead to an increase of the permeability and the void saturation in the porous asphalt mixtures.
\end{abstract}

Key words : porous asphalt mixture; unidirectional permeability; multidirectional permeability; directional difference; permeameter; void saturation 


\section{Introduction}

Porous asphalt mixtures have been widely used in asphalt pavements due to their excellent performance in skid resistance, noise reduction, rain drainage and anti-glare. Their performance is attributed to rich connected voids within the porous asphalt mixture (Colwill et al. 1999; Huber 2000; Fwa et al. 2001; Tan et al. 2003; Yong et al. 2013). In a design process of the porous asphalt mixtures, two methods are commonly adopted to ensure the connected voids: one is to control total air void content or interconnected air voids (Alvarez et al. 2011); the other method is to measure the permeability property and use it to reflect the connectivity of the internal air voids in the porous asphalt mixtures (Alvarez et al. 2011; Chen et al. 2015). The total air void content can reflect the overall porosity of the porous asphalt mixture, however the permeability can characterize not only the overall porosity but the void connectivity and size which are closely related with the drainage function of the mixture. Therefore, it is crucial to verify the permeability of the porous asphalt mixture during its design to ensure the functions of drainage, anti-skid and noise reduction.

The permeability property (e.g., permeability coefficient) is usually assumed directional independent when tested in laboratory or in the field. However asphalt mixture is a heterogeneous and anisotropic material (Zhang et al. 2011, 2012, 2014). Its interior void structure may have directional variation (Gu et al. 2015a, 2015b). This variation leads to a directional dependence for the water permeability of the mixtures (Masad et al. 1999). Al-Omari and Masad (2004) utilized a semi-implicit method for pressure-linked equations (SIMPLE) finite difference scheme to simulate the water flow in an asphalt mixture and calculated the longitudinal, transverse and vertical permeability coefficients for eight types of asphalt mixture. They found little differences between longitudinal and lateral permeability coefficients, but the vertical permeability coefficient is much less than that in longitudinal and lateral directions. Macro-scale modeling study conducted by Hunter and Airey (2005) also showed that the direction of the water flow in asphalt mixtures is mainly parallel to the road surface and little flow has occurred in the vertical plane (parallel to compaction direction). Kutay et al. (2007) adopted X-ray Computational Tomography (CT) and mathematical morphology-based techniques to establish three-dimensional pore structures in densegraded asphalt mixtures. They also simulated the water flow in the void structure using a three-dimensional lattice Boltzmann (LB) fluid flow model. It was found that the radial permeability coefficient was higher than the vertical permeability coefficient by two orders of magnitude. However the permeability coefficients in the two orthogonal directions of the horizontal plane were comparable within a $50 \%$ confidence interval. 
To address the anisotropic properties of the permeability, some researchers investigated the directional dependence of the permeability for the porous asphalt mixtures. Masad et al. (2007) analyzed the directional dependence of the permeability coefficient and the connected voids in asphalt mixtures based on the images scanned by X-ray CT. The results showed that the connected voids provided more drainage channels in the horizontal direction rather than that in the vertical direction. The ratio of the horizontal to the vertical permeability coefficient is from 8.35 to 11.40 for dense asphalt mixtures and from 1.65 and 4.02 for porous asphalt mixtures. Gruber et al. (2012) obtained the three-dimensional internal structure of a porous asphalt mixture using Xray $\mathrm{CT}$ and modelled the water flow in a three-dimensional void structure. They found that the difference of permeability coefficient in three directions was about 50\%, which differed from the results by Masad et al. Chen et al. (2017) measured the permeability in twelve directions in the vertical plane of the porous asphalt mixture. The ratios of maximum and minimum permeability were found in a range of 1.4 to 1.7 for porous mixture with the nominal maximum aggregate size of $13.2 \mathrm{~mm}$.

It is concluded from the literature review that: (1) little research effort has been spent on the directional permeability study of the porous asphalt mixtures compared to that of the dense graded asphalt mixtures. Researchers have drawn different conclusions on the magnitude of the permeability properties in different directions; (2) the existing studies are mainly based on numerical modelling and X-Ray CT, while less on direct laboratory measurement to determine the directional dependence of the permeability for porous mixtures; (3) although the author (Chen et al. 2017) has recently investigated on the permeability anisotropy in the vertical plane of porous mixtures, the permeability anisotropy in the horizontal plane (perpendicular to compaction direction) and the effect of air void content on the permeability anisotropy are still unclear; (4) a systematical study is needed to evaluate the multidirectional permeability of the porous asphalt mixtures since the unidirectional permeability is inappropriate to characterize the directional dependence.

This study aims to evaluate the directional dependency of the permeability for the porous asphalt mixtures. The specific objectives of this research include:

(1) An experimental method will be designed to measure the permeability in different directions in both vertical and horizontal planes of the porous mixtures. The unidirectional constant water-head permeameter will be used in this method to measure the permeability in different directions for the porous mixtures.

(2) This study will analyze the influences of air void content and nominal maximum aggregate size of asphalt mixtures on the directional difference of permeability. 
(3) Based on the actual water flow process in pavement structure, this study will employ a permeameter device to measure the multidirectional permeability and analyze the effects of the air void content and aggregate size on water saturation in voids.

\section{Laboratory test on porous mixtures’ permeability}

\subsection{Test method, device and materials}

The permeability of a porous asphalt mixture in twenty-four directions were tested including twelve directions in the horizontal plane (perpendicular to compaction direction) and twelve directions in the vertical plane (parallel to compaction direction), as shown in a flow chart in Fig. 1. The reason of measuring the permeability in 12 directions in both vertical and horizontal planes is to evaluate and understand the spatial permeability characteristics and directional dependence of the mixtures in three dimensional conditions.

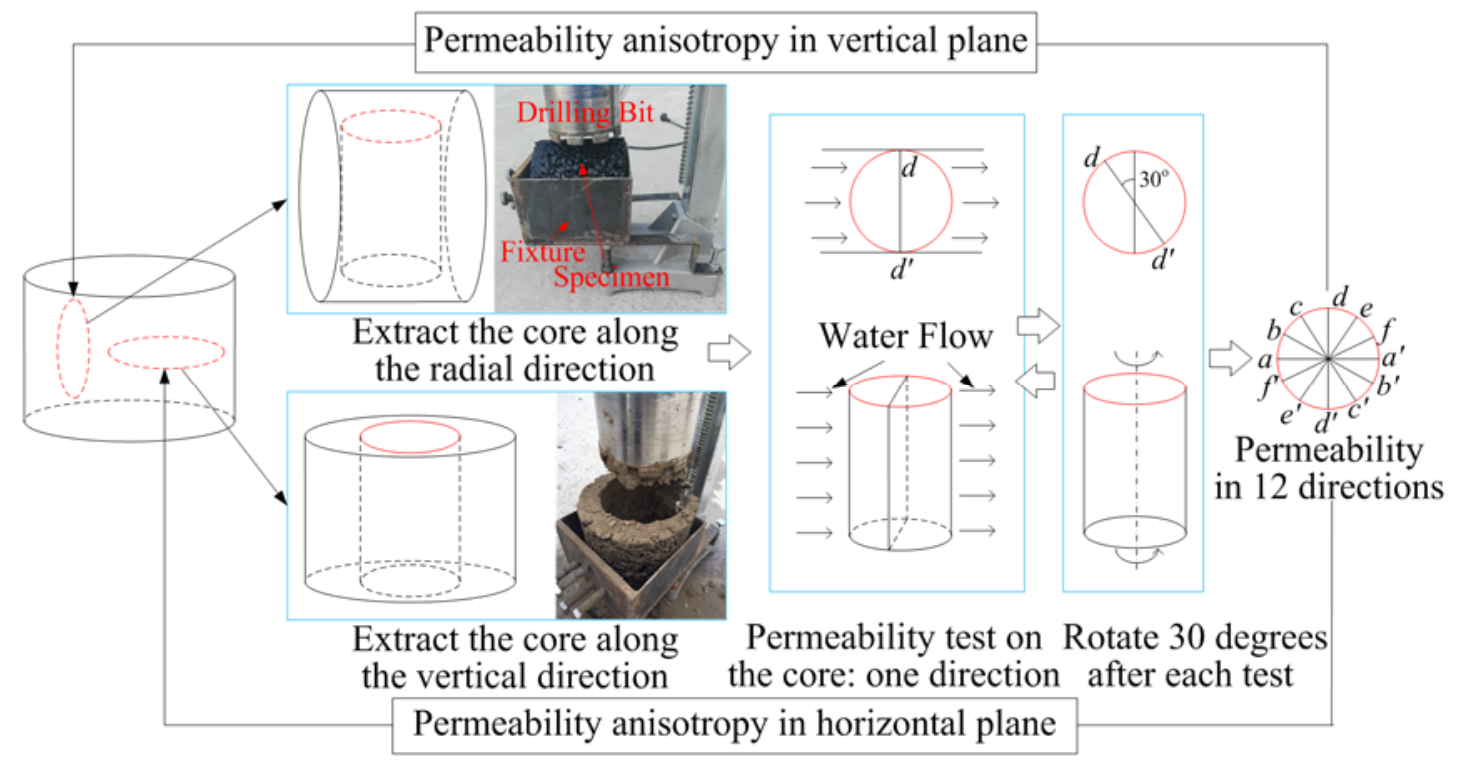

Fig. 1. Flow chart of permeability test in twenty-four directions (Chen et al. (2017))

The specific testing steps are described as follows:

(1) Six replicates of one open graded friction course (OGFC) asphalt were compacted using a gyratory compactor to obtain the asphalt mixture samples with 120 $\mathrm{mm}$ in height and $150 \mathrm{~mm}$ in diameter.

(2) Three of the six cylindrical samples were cored along the radial direction and then the two ends were cut to flat surfaces to obtain three small cylindrical samples with $100 \mathrm{~mm}$ in height and $100 \mathrm{~mm}$ in diameter. Note that this $100 \mathrm{~mm} \times 100 \mathrm{~mm}$ are the maximum dimensions for the cylindrical samples that can be cored and cut in the radial direction.

(3) The permeability was tested using a unidirectional constant water-head 
permeameter, as shown in Fig. 2. The water head was set as $500 \mathrm{~mm}$. The volume $V$ (ml) for the water passing the sample was measured during a time period of $t$ (s) and the permeability is quantified by Equation 1. The water flow direction was marked on the lateral end of the sample.

$$
\text { Permeability }=\frac{V}{t}
$$

It should be noted that the water head of $500 \mathrm{~mm}(\approx 18.75 \mathrm{~m} \times 2.5 \%)$ was calculated based on the water flow through the interconnected air voids along the transversal direction of a pavement with a width of $18.75 \mathrm{~m}$ and a transversal slope of $2.5 \%$. The permeameter was developed to measure the permeability of the core along different directions by rotating the core sample. Equation 1 is the water flow rate which is used as a parameter to quantify the permeability of the asphalt mixtures at the given water head and sample dimensions. Note that Equation 1 is not equivalent to the permeability coefficient which is used in the Darcy law that is valid only for a laminar flow, however the permeameter and the permeability parameter in Equation 1 are applicable to quantify the permeability for different asphalt mixture samples no matter if the water flow is a laminar flow or a turbulent flow in the samples.

(4) The sample was removed from the permeameter and rotated for 30 degrees from the previously marked direction along its vertical axis. Then the sample was installed back to the permeameter and tested the permeability in this new direction.

(5) Step (3) and Step (4) were repeated for eleven times and a total of twelve permeability in twelve different directions were obtained to evaluate the directional dependence of the permeability in the vertical plane.

(6) The remaining three samples obtained from Step (1) were cored along the vertical direction and their two ends were also cut flat to obtain three small cylindrical samples with $100 \mathrm{~mm}$ in height and $100 \mathrm{~mm}$ in diameter. Repeating Step (3) and Step (4) for twelve times results in the permeability measurements in twelve directions in the horizontal plane.

It is noted that, for the small cylindrical samples cored along the radial direction of the large samples (in Step 2), the permeability tests yield twelve permeability in the vertical plane of the large samples. Similarly, for the small cylindrical samples cored along the vertical direction of the large samples (in Step 6), the permeability tests yield twelve permeability in the horizontal plane of the large samples. 


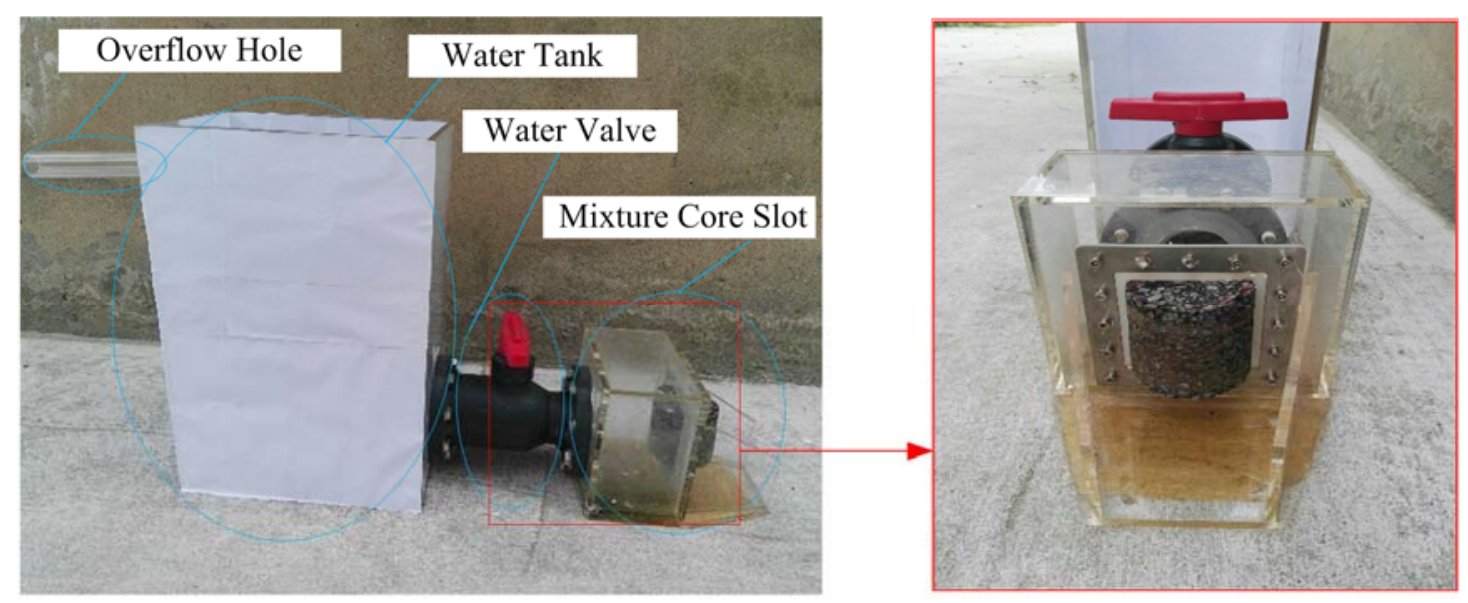

Fig. 2. Configuration of unidirectional constant water-head permeameter

(Chen et al. (2017))

Table 1 shows the aggregate gradation and asphalt binder content for the eight different asphalt mixtures. In Table 1, No.1, No.2, No.3, and No.4 are the OGFC-13 mixtures with design air void content of $17 \%, 19 \%, 21 \%$, and $23 \%$, respectively. The No.5, No.6, No.7, and No.8 are the OGFC-10 mixtures with design air void content of $17 \%, 19 \%, 21 \%$, and $23 \%$, respectively. The air void content of the OGFC samples was determined using the maximum theoretical density and the bulk density by volume testing method. The OGFC samples used in the permeability test have the measured void contents which are within $0.5 \%$ variance from the corresponding design void content in Table 1. OGFC-13 and OGFC-10 mixtures have the nominal maximum aggregate sizes of $13 \mathrm{~mm}$ and $10 \mathrm{~mm}$, respectively. The asphalt binder was a highviscosity modified asphalt. Basalt was chosen as the aggregate and limestone powder was selected as the filler.

Table 1 Aggregate degradation and asphalt content of OGFC-13 and OGFC-10

\begin{tabular}{cccccccccccccc}
\hline \multirow{2}{*}{ No. } & \multicolumn{1}{c}{ Mass percentage (\%) passing the below sieve size $(\mathrm{mm})$} & AC & VV & OGFC \\
& 16 & 13.2 & 9.5 & 4.75 & 2.36 & 1.18 & 0.6 & 0.3 & 0.15 & 0.075 & $(\%)$ & $(\%)$ & type \\
\hline 1 & 100 & 92.0 & 66.4 & 28.1 & 16.7 & 13.1 & 10.3 & 7.4 & 6.3 & 4.9 & 4.8 & 17 & \\
2 & 100 & 91.0 & 62.5 & 25.5 & 16.4 & 12.9 & 10.2 & 7.3 & 6.2 & 4.9 & 4.7 & 19 & OGFC \\
3 & 100 & 90 & 60.1 & 21.1 & 13.7 & 10.7 & 8.5 & 6.3 & 5.5 & 4.3 & 4.5 & 21 & -13 \\
4 & 100 & 95 & 77.5 & 26.1 & 13.5 & 10.2 & 7.9 & 5.8 & 5.1 & 4.1 & 4.4 & 23 & \\
\hline 5 & 100 & 100 & 98.1 & 60.9 & 18.1 & 13.6 & 10.7 & 7.8 & 6.7 & 5.2 & 5.0 & 17 & \\
6 & 100 & 100 & 97.4 & 57.4 & 17.2 & 13.2 & 9.1 & 7.1 & 5.5 & 4.6 & 4.8 & 19 & OGFC \\
7 & 100 & 100 & 97.5 & 57.2 & 15.9 & 12.8 & 8.4 & 5.9 & 5.1 & 4.2 & 4.7 & 21 & -10 \\
8 & 100 & 100 & 96 & 53.2 & 15.1 & 12.4 & 7.4 & 6.0 & 4.7 & 3.2 & 4.5 & 23 & \\
\hline
\end{tabular}

$*_{\mathrm{AC}}$ is asphalt content. $\mathrm{VV}$ is design air void content.

\subsection{Test results}

The permeability in the twenty-four directions were measured for the eight 
different OGFC mixtures shown in Table 1. Fig. 3 shows the permeability of the OGFC13 mixtures with different air void contents in twelve directions in the vertical plane and the horizontal plane, respectively. The permeability for OGFC-10 are shown in Fig. 4.

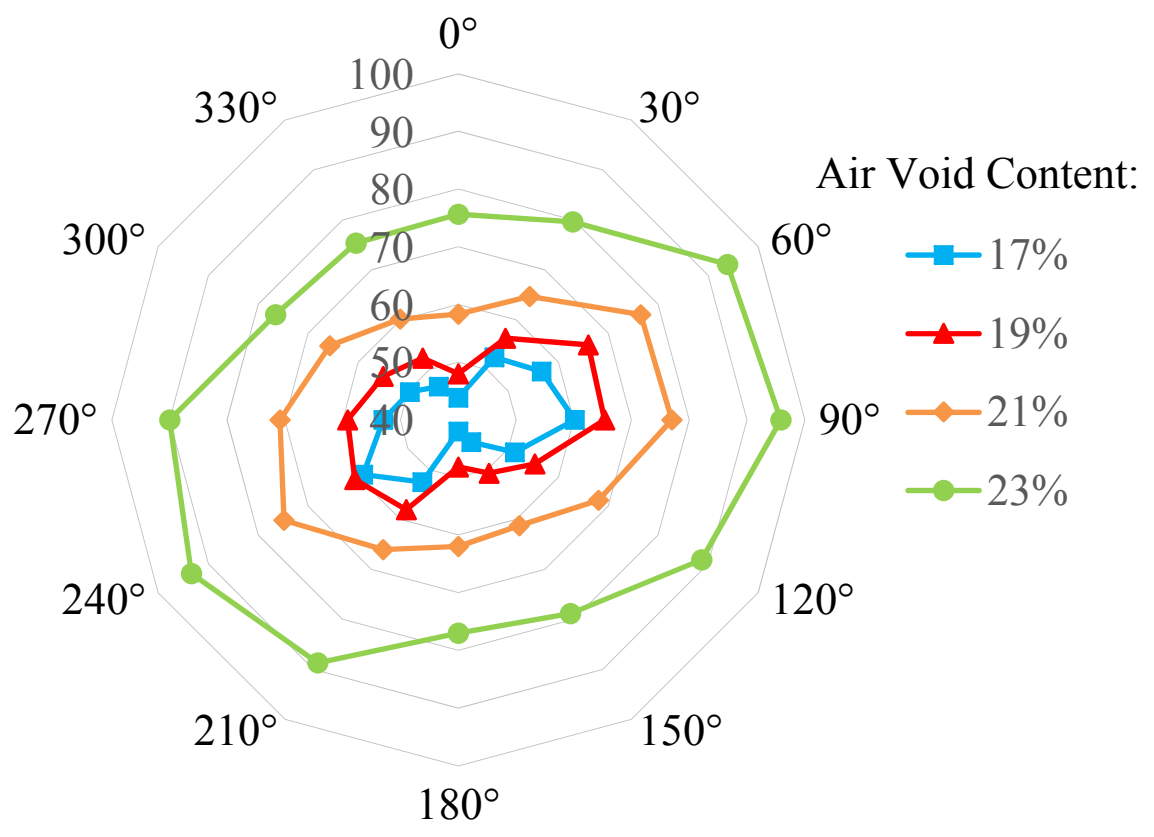

(a) Vertical plane

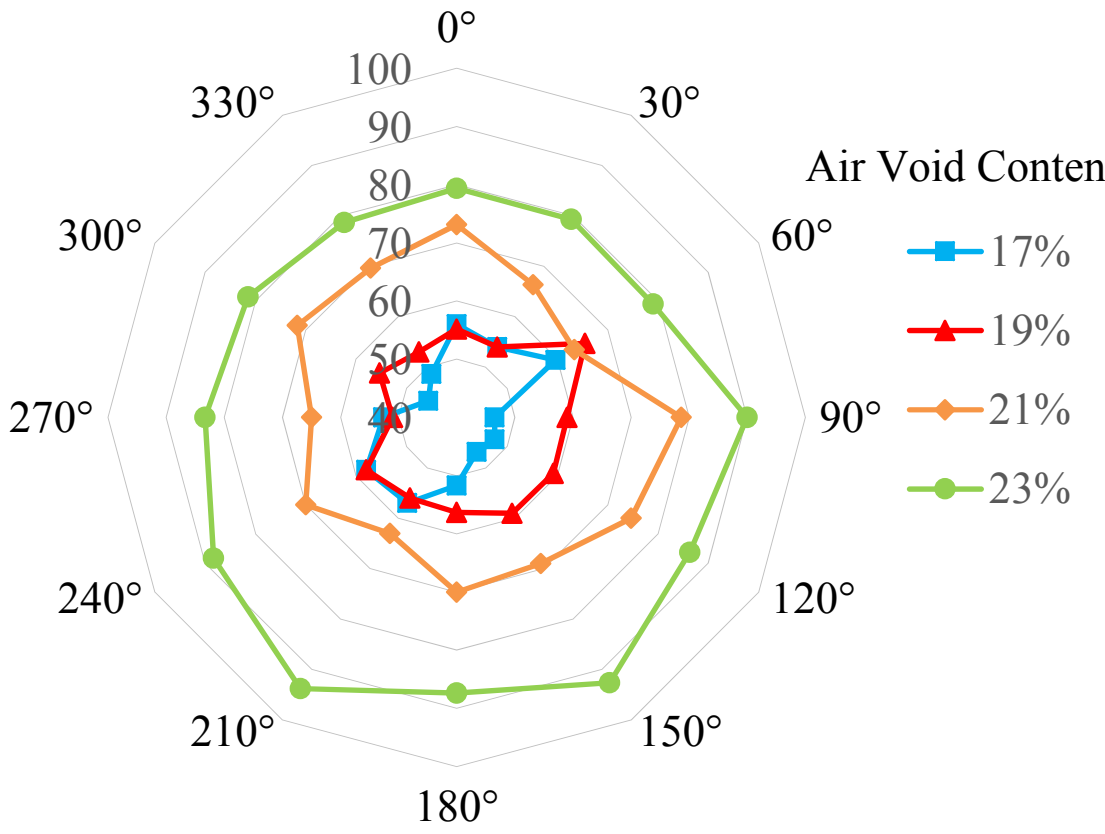

(b) Horizontal plane

Fig. 3. Permeability (ml/s) of OGFC-13 mixtures with different air void contents in twelve directions in the vertical plane (a) and horizontal plane (b)

From Fig. 3(a) and Fig. 4(a), it can be found that the permeability of the OGFC mixture is directionally dependent in the vertical plane. The minimum permeability 
occurs in the vertical direction $\left(0^{\circ}\right.$ and $180^{\circ}$ directions) and the maximum permeability appears in the direction between $60^{\circ}$ and $90^{\circ}$ apart from the vertical axis. This means that the void connectivity is higher in the horizontal direction than that in the vertical direction. Moreover, Figs. 3(a) and 4(a) illustrate that the permeability distribution in vertical plane changes from a " $\infty$ " shape to an ellipse shape when the air void content increases from $17 \%$ to $23 \%$, indicating a less directional dependence at a higher air void content.

Furthermore, in the vertical plane, the permeability in $0^{\circ}$ direction differs from that in $180^{\circ}$ directions. The same results were observed between the opposite directions, e.g., $30^{\circ}$ and $210^{\circ}, 60^{\circ}$ and $240^{\circ}, 90^{\circ}$ and $270^{\circ}, 120^{\circ}$ and $300^{\circ}, 150^{\circ}$ and $330^{\circ}$, etc. Thus it demonstrates that permeability are different in the two opposite directions.

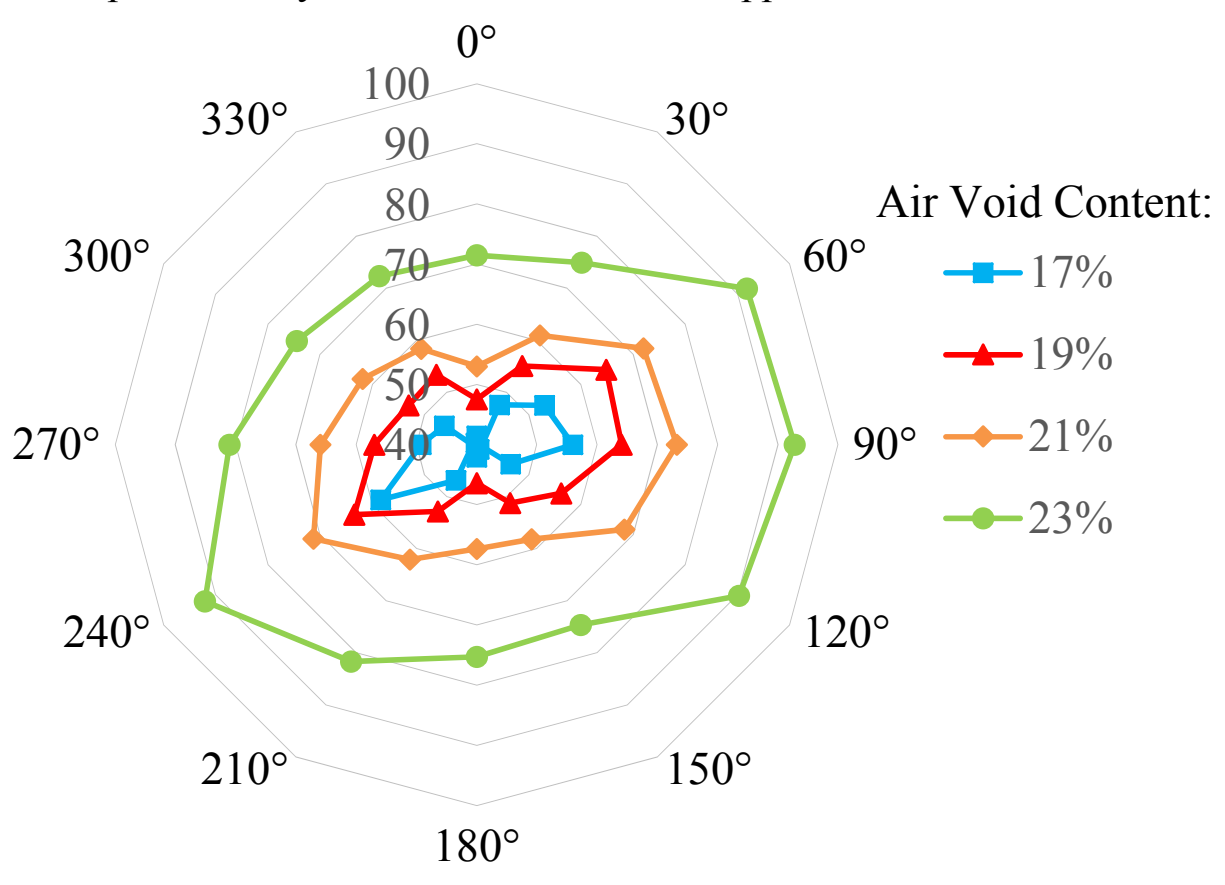

(a) Vertical plane 


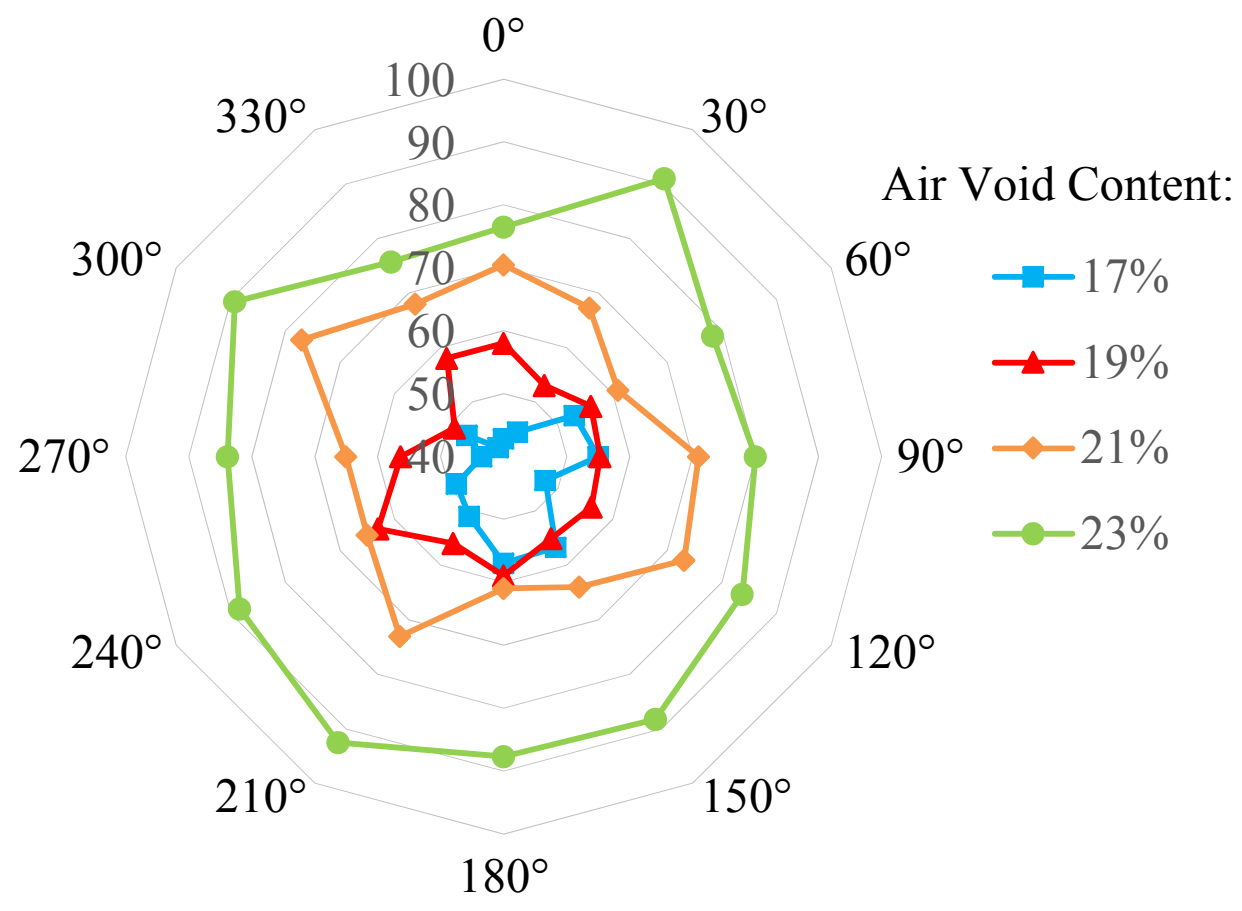

(b) Horizontal plane

Fig. 4. Permeability (ml/s) of OGFC-10 mixtures with different air void contents in twelve directions in the vertical plane (a) and horizontal plane (b)

It can be seen from Fig. 3(b) and Fig. 4(b) that the permeability also depends on the directions in the horizontal plane. Furthermore, an OGFC mixture with a higher air void content shows less directional dependence for the permeability. However, the relationship between the permeability and the directional angle in the horizontal plane is less significant than that in the vertical plane.

\subsection{Influence of air void content and aggregate size on permeability}

Using the permeability in Fig. 3 and Fig. 4, permeability anisotropy $(P A)$ is defined as follows:

$$
P A=\frac{P_{\max }-P_{\min }}{P_{\min }} \times 100 \%
$$

where $P_{\max }$ and $P_{\min }$ are the maximum and minimum permeability, respectively, of the twelve directions in horizontal or vertical plane.

The relationship between $P A$ and air void content and aggregate size are shown in Fig. 5. It can be found that for the same mixture type $P A$ decreases with air void content in both horizontal plane and vertical plane. At the same air void content, the OGFC-13 mixture has a smaller $P A$ compared with the OGFC-10 mixture. Therefore, $P A$ decreases with the increase of the nominal maximum particle size.

It can also be seen from Fig. 5 that the $P A$ in the vertical plane is higher than that 
in the horizontal plane for the OGFC mixtures with the same air void content. As a result, permeability anisotropy of porous asphalt mixtures is more significant in the vertical plane than that in the horizontal plane. However, the permeability anisotropy in the horizontal plane is still very high (greater than 20\%) that should be taken into account in permeability evaluation.

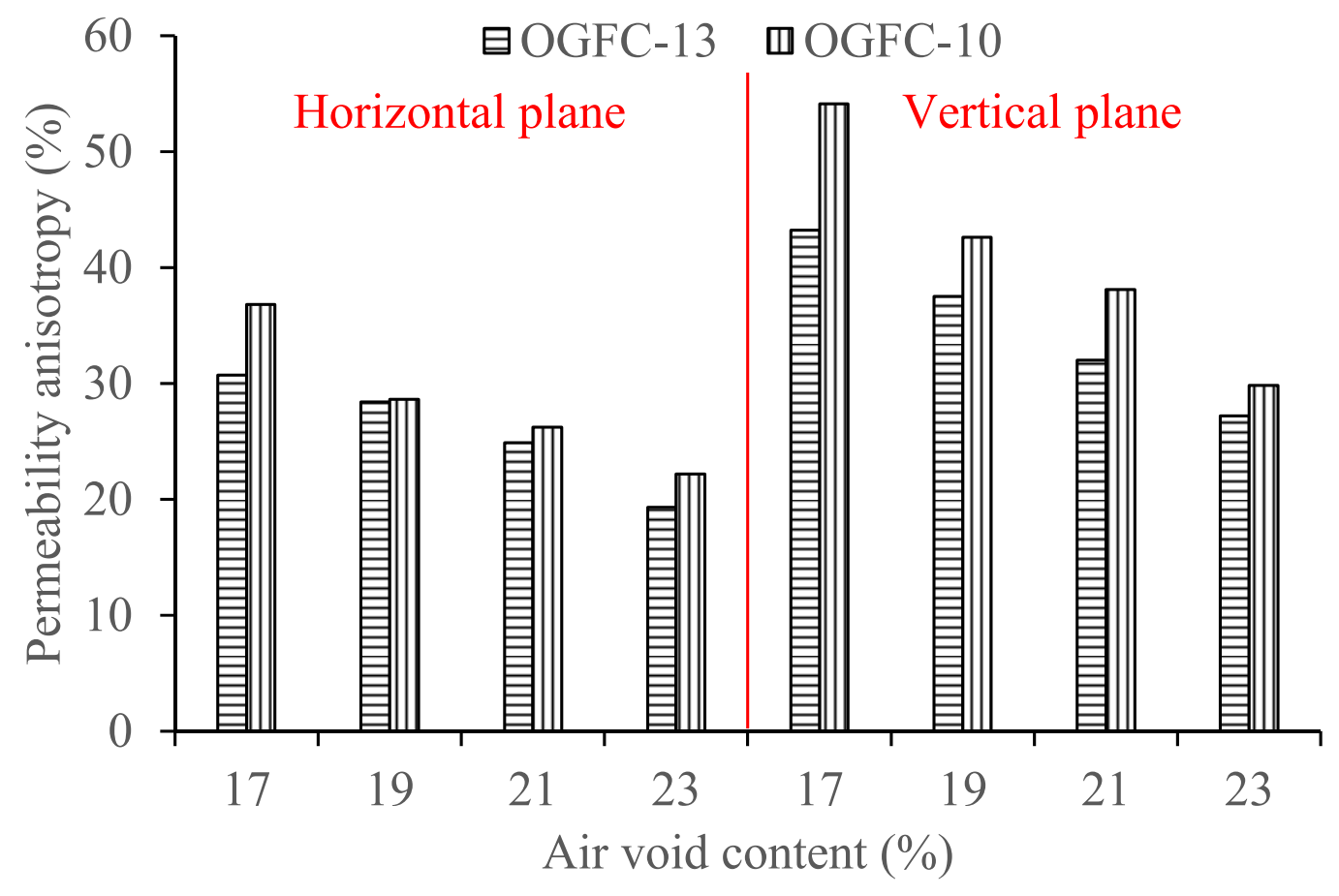

Fig. 5. Permeability anisotropy vs air void content for OGFC-10 and OGFC13

\section{Laboratory test for multidirectional permeability}

\subsection{Testing device}

The study in Section 3 has shown that permeability is anisotropic, having different values in different directions. Thus it becomes inappropriate to adopt a single permeability (a permeability measured in one direction) to evaluate the anisotropic permeability of porous asphalt mixtures. For this reason the flow process of water runoff in a real pavement layer structure was analyzed as shown in Fig. 6 (Chen et al. 2015). Then a multidirectional constant water-head permeameter (Fig.7a) was designed to simulate the process of water infiltrating into an OGFC slab specimen. The dimension for the OGFC slab is $300 \mathrm{~mm} \times 300 \mathrm{~mm} \times 50 \mathrm{~mm}$. The design of the permeameter device was explained in detail in the previous studies (Chen et al. 2015; Chen et al. 2016). As seen in Fig. 7(b), it was fabricated using Plexiglas including a water tank, a horizontal slope adjustment system, a surface water collection tank, an internal permeating water collection tank and a support base. 


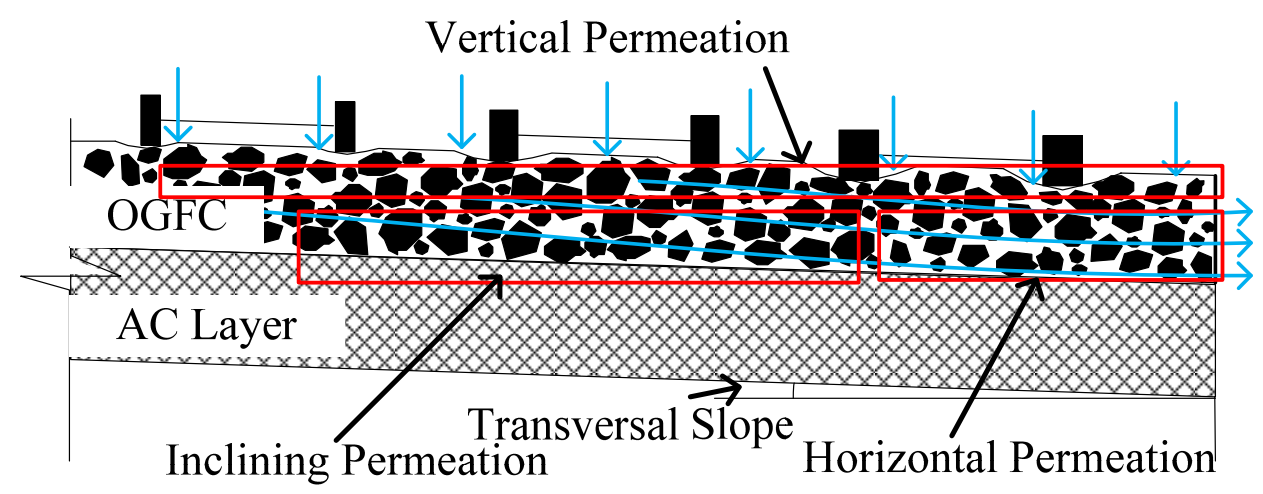

Fig. 6. Flow of water runoff in OGFC pavement (Chen et al. 2015)

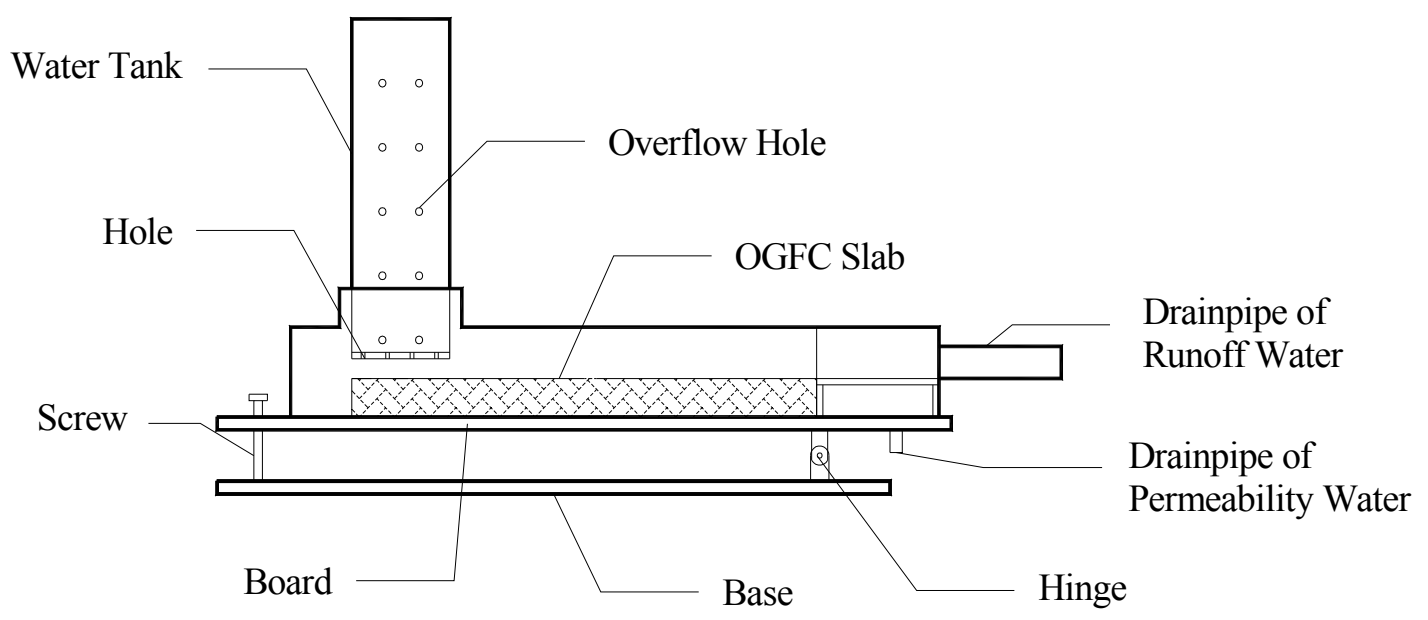

(a) Schematic diagram

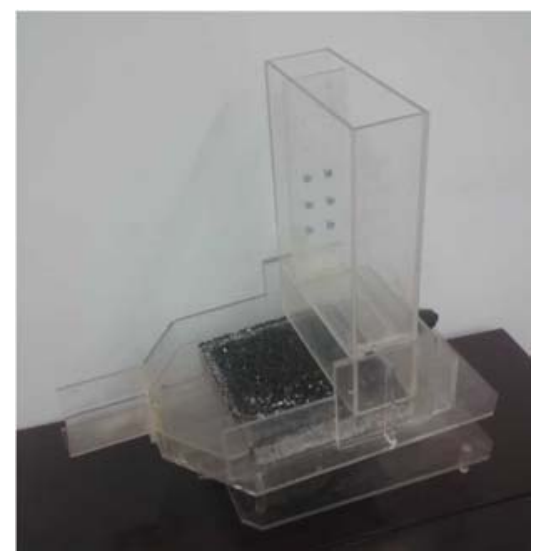

(b) Photograph of permeameter

Fig. 7. Multidirectional constant water-head permeameter device (Chen et al. (2017))

As shown in Fig. 7, the water head in the water tank of the permeameter can be adjusted to simulate the different intensity of rainfall. The water head was set at $9 \mathrm{~cm}$, $14 \mathrm{~cm}, 19 \mathrm{~cm}, 24 \mathrm{~cm}$ and $29 \mathrm{~cm}$, corresponding to the rainfall intensity of $12.5 \mathrm{ml} / \mathrm{s} \cdot \mathrm{m}^{2}$, 
$15.7 \mathrm{ml} / \mathrm{s} \cdot \mathrm{m}^{2}, 21.2 \mathrm{ml} / \mathrm{s} \cdot \mathrm{m}^{2}, 24.7 \mathrm{ml} / \mathrm{s} \cdot \mathrm{m}^{2}$ and $27.1 \mathrm{ml} / \mathrm{s} \cdot \mathrm{m}^{2}$ respectively. The angle between the board and the permeameter base can be adjusted by rotating the hinge shown in Fig. 7(a) to simulate different transverse slope of a pavement, where in this study two slopes (i.e., $1.0 \%$ and $1.5 \%$ ) were selected.

When the water in the tank flows onto the OGFC slab through the overflow holes, it will firstly permeate into the slab, and then flow along the slope. Some of the water will permeate out of the slab from the slab's lateral surface and this part of water is termed as lateral permeating water. When the air void content of the slab is relatively small or the dripping rate of the water tank is very high (simulating a high rainfall intensity), surface runoff water may occur on the surface of the OGFC slab. The lateral permeating water and the runoff water can be collected and measured separately by the permeameter.

The permeability of the OGFC slab were tested by the new permeameter according to the following procedures:

(a) The top surface of the OGFC slab with a dimension of $300 \mathrm{~mm} \times 300 \mathrm{~mm}$ was selected as the drainage surface for runoff water. One of the lateral surfaces with a dimension of $300 \mathrm{~mm} \times 50 \mathrm{~mm}$ was chosen as the internal drainage surface for the lateral permeating water, and all other lateral surfaces were sealed with wax. The slab was then placed on the board of the permeameter and the unsealed lateral surface is positioned closely to the water collecting tank. After that, the hinge was rotated to adjust the permeameter to meet the requirement of the intended slope.

(b) Water head was set by selecting an overflow hole on the clapboard of the water tank, and screws were used to plug all the other overflow holes beneath the selected one. Water was added into the tank until the set height was reached. Start timing and collecting the surface runoff water and the lateral permeability water for 120 seconds when the water head becomes stable for over 1 minute. This procedure was repeated for the five different water head levels.

(c) When surface runoff started to occur, the water head was recorded and used as the water head for determining the multidirectional permeability. At this water head the water flowing from the lateral surface was measured as $V_{1}(\mathrm{ml})$. The multidirectional permeability $M P(\mathrm{ml} / \mathrm{s})$ is calculated by Equation (3). It should be noted that at this situation the internal voids of the OGFC mixture have not completely occupied by water. Further discussion about this saturation state will be introduced in this paper.

$$
M P=\frac{V_{1}}{120}
$$

\subsection{Directional sensitivity of MP}


As found in Section 3.2, unidirectional permeability of porous asphalt mixtures varies in different directions, thus $M P$ should be adopted to evaluate the permeability of the mixtures. However, it is unknown if $M P$ varies when different surfaces of the testing mixture slab are used as the permeating surfaces. Therefore, a slab with No.5 gradation in Table 1 was fabricated by roller compactor, as shown in Fig. 8. The MP of the slab was tested using surface No. 5 (top face in Fig. 8) and surface 6 (bottom face in Fig. 8) as the top permeating surface. The lateral surfaces No. 1, 2, 3, and 4 were used as the lateral permeating faces, respectively. The same transversal slope of $1 \%$ was used for all tests and the results of $M P$ are shown in Fig. 9.

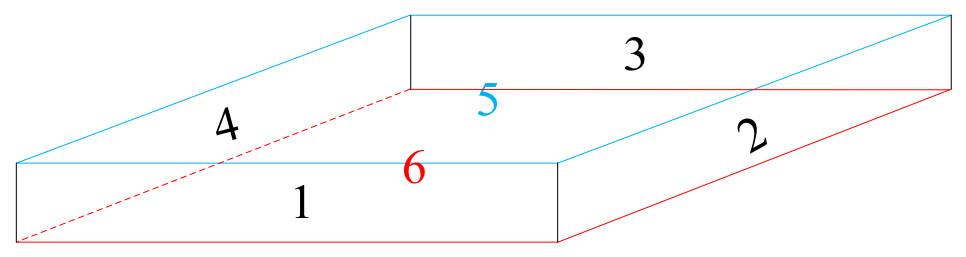

Fig. 8. Surface Number of the Asphalt Mixture Slab

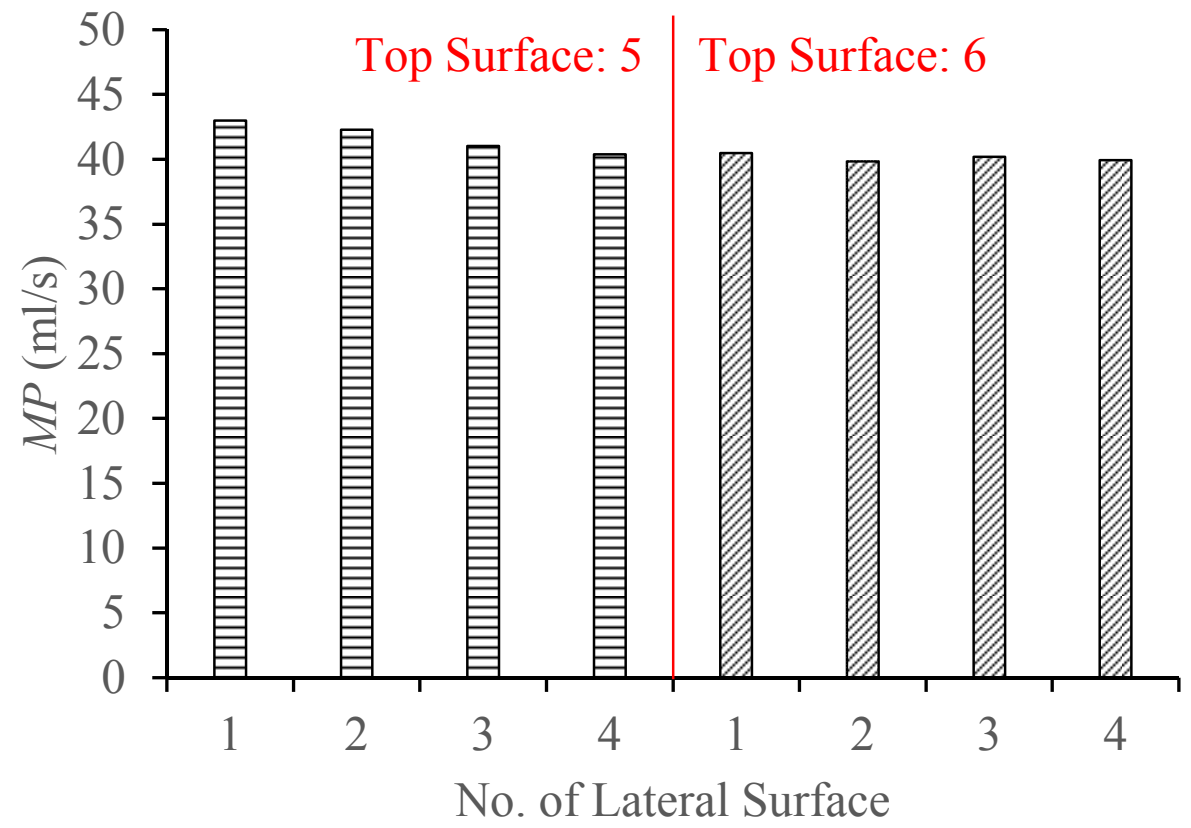

Fig. 9. Multidirectional permeability when different OGFC mixture slab surfaces used as permeating faces

Fig. 9 shows little differences for $M P$ when using surfaces No. 5 or No. 6 as the top permeating surface or using lateral faces No. 1, 2, 3 or 4 as the lateral permeating surfaces. The difference between the maximum $M P(43 \mathrm{ml} / \mathrm{s})$ and the minimum value $(40 \mathrm{ml} / \mathrm{s})$ is within $10 \%$. This difference is much smaller than that obtained by the unidirectional permeameter. As a result, the $M P$ tested by the directional permeameter 
developed in this study is not affected by water flow directions, hence it can accurately evaluate the permeability of porous asphalt mixtures.

\subsection{Test results}

(1) Multidirectional Permeability $(M P)$

$M P$ values were measured for eight types of asphalt mixtures shown in Table 1 with the multidirectional permeameter at two transversal slopes, namely $1 \%$ and $1.5 \%$. The results are shown in Fig. 10.

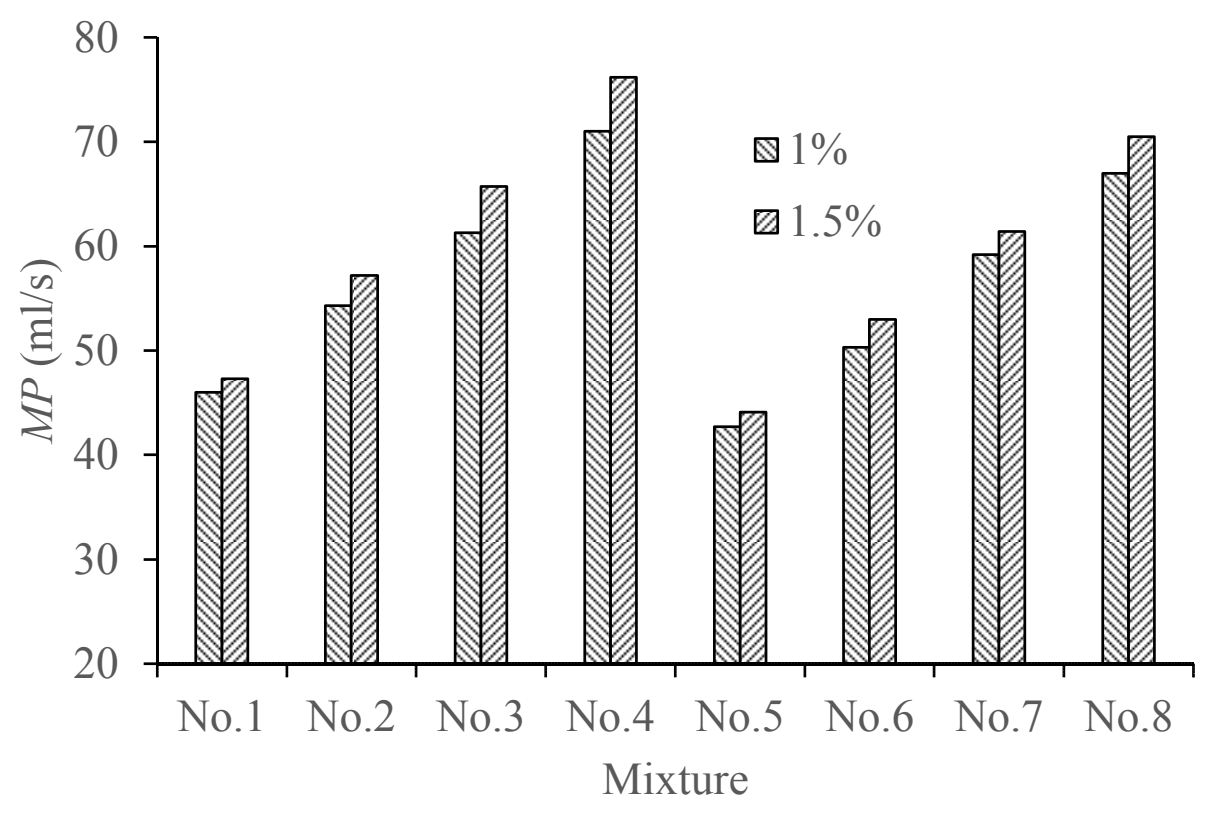

Fig. 10. Multidirectional permeability for the eight different porous asphalt mixtures (shown in Table 1) at two transversal slopes (1\% and $1.5 \%)$.

Fig. 10 shows that the $M P$ of the OGFC mixture is higher for the mixtures with a higher air void content or at a larger transverse slope. Compared with transverse slope, air void content has a higher impact on the $M P$. In addition, the transverse slope has bigger impact on the $M P$ of the No.4 and No.8 mixtures with an air void content of $23 \%$ than that of No.1 and No.5 mixtures with an air void content of $17 \%$.

Fig. 10 also indicates that $M P$ are different for OGFC-13 and OGFC-10 mixtures even though the air void content remains the same. For example, MP of OGFC-13 mixture (No. 1) are higher than that of OGFC-10 mixture (No. 5) though both have an air void content of $17 \%$. Therefore, increasing the nominal maximum size of aggregate is beneficial to improving asphalt mixtures' permeability.

(2) Void Saturation

Void saturation is defined as the ratio of the volume of the water in the voids to the 
total volume of the voids. In the $M P$ test the water flowing from the lateral surface was collected when the water tank was removed. The volume of this part of water was measured as $V_{2}$. The slab was then removed from the permeameter when there was no water flowing out, and its wet mass was measured as $m_{\mathrm{w}}$. Void saturation of the porous asphalt mixtures $(S)$ can be obtained by Equation (4).

$$
S=\frac{V_{w}}{V_{a}} \times 100 \%=\frac{V_{2}+\frac{m_{w}-m_{d}}{\rho}}{V_{3} \cdot V V} \times 100 \%=\frac{\rho V_{2}+m_{w}-m_{d}}{\rho \cdot V_{3} \cdot V V} \times 100 \%
$$

where, $V_{w}$ is the volume of the water in voids; $V_{a}$ is the total volume of the voids; $m_{d}$ is the dry mass before the $M P$ testing; $V_{v}$ is the air void content; $\rho$ is water density; $V_{3}$ is the volume of slabs, which can be calculated by its dimensions.

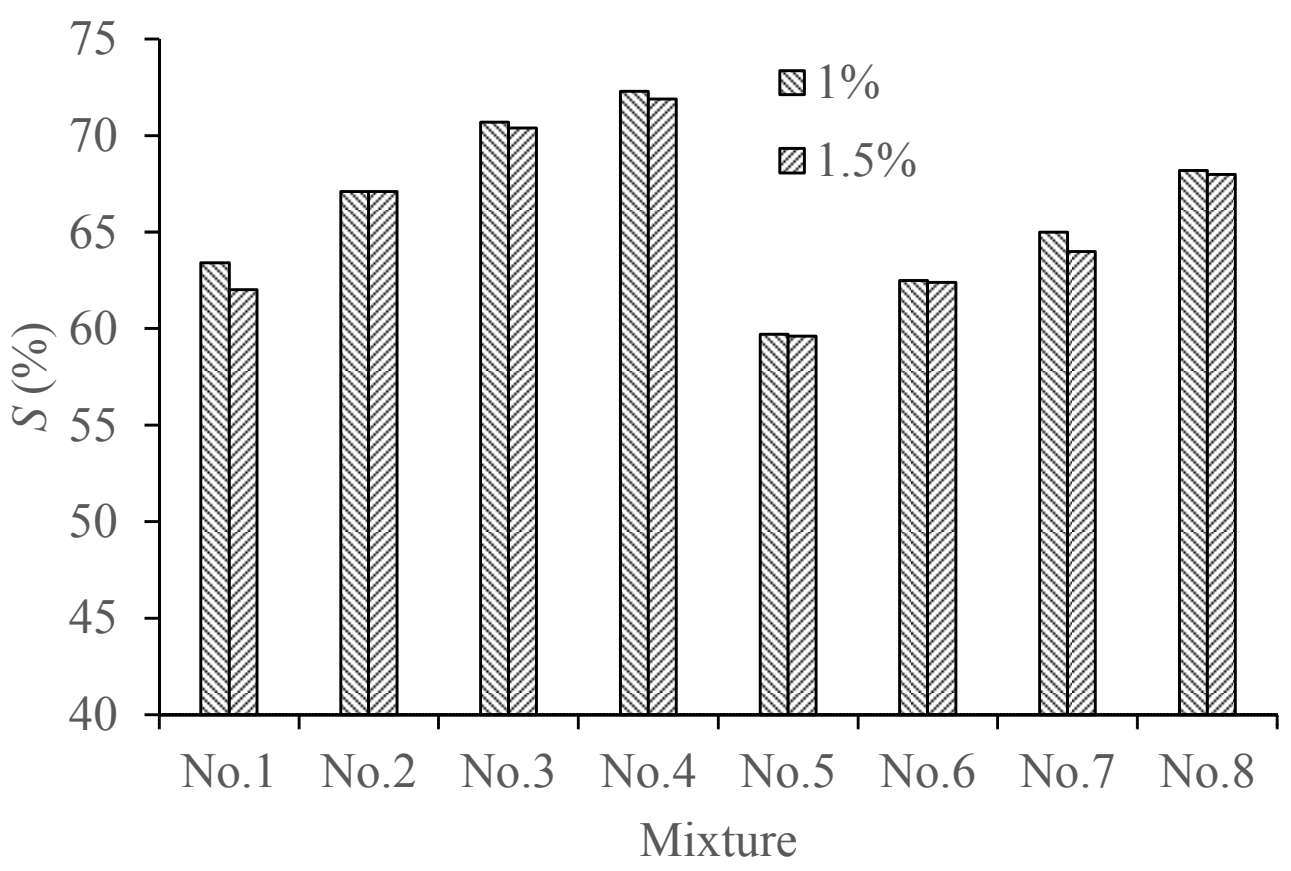

Fig. 11. Void saturations for the eight different porous asphalt mixtures (Table 1)

Fig. 11 shows that the void saturation is less than 100 percent for all porous mixtures, which indicates that the internal voids of the porous asphalt mixtures were not completely occupied by water (unsaturated) when runoff occurs on the top surface of the samples. The volume of water did not exceed 75 percent of the voids volume for the tested porous asphalt mixture.

It can also be observed from Fig. 11 that void saturation becomes higher with an increasing air void content (from No. 1 to 4 or from No. 5 to 8), but the transversal slope has little effect on the void saturation. Moreover, at the same air void content, the void saturation of OGFC-13 is slightly higher than that of OGFC-10. As a result, 
increasing the air void content or the maximum aggregate size can contribute to the utilization of voids in storing water in the porous asphalt mixtures.

The future work following this study is to evaluate the effect of the rainfall intensity on the water saturation and water flow status in the porous asphalt mixtures, as well as to verify the repeatability of the directional permeameter by using a larger size for the samples. The implementation of the directional dependence of the permeability in porous asphalt mixture design will also be considered in the future studies.

\section{Conclusions}

(1) The permeability of porous asphalt mixture was demonstrated distinct in different directions of the vertical plane (parallel to compaction direction) as well as in different directions of the horizontal plane (perpendicular to compaction direction). However the difference of permeability is more significant in the vertical plane than that in the horizontal plane.

(2) In the vertical plane, the minimum permeability occurs in the vertical direction and the maximum value occurs in the horizontal direction. In the horizontal plane, the permeability are different in different directions, but has no obvious relationship with horizontal directions.

(3) Increasing air void content or nominal maximum particle size of the porous asphalt mixtures can reduce the directional difference of the permeability for the porous asphalt mixtures.

(4) The developed multidirectional permeability tester can simulate the real water flow process and the slope of a pavement, and control rainfall intensity. The new permeameter was used to test multidirectional permeability of porous asphalt mixtures, which overcomes the problem of directional difference of unidirectional permeability.

(5) The internal voids of the porous asphalt mixtures are not completely occupied by water when runoff occurs on the top surface. Increasing the air void content or the aggregate size will lead to an increase of the multidirectional permeability and the void saturation of the porous mixtures.

\section{Acknowledgments}

The research presented herein was sponsored by the National Natural Science Foundation of China (No. 51208178) and the Fundamental Research Funds for the Central Universities (No. 2015B17014).

\section{Reference}


Colwill, D. M., Bowskill, G. J., Nicholls, J. C., and Daines, M. E. (1993). "Porous asphalt trials in the United Kingdom.” Transp. Res. Record, 1427(1), 13-21.

Huber, G. (2000). "Performance survey on open-graded friction course mixes." National Cooperative Highway Research Program (NCHRP) Synthesis of Highway Practice 284, National Academies, Washington, DC.

Fwa, T. F., Tan, S. A., Chuai, C. T., and Guwe, Y. K. (2001). "Expedient permeability measurement for porous pavement surface.” Int. J. Pavement Eng., 2(4), 259-270.

Alvarez, A. E., Martin, A. E., and Estakhri, C. (2011) "A review of mix design and evaluation research for permeable friction course mixtures." Constr. Build. Mater, 25(3), 1159-1166.

Zhang, Y., Luo, R., and Lytton, R. L. (2011). "Microstructure-based inherent anisotropy of asphalt mixtures”. J. Mater. Civ. Eng., 23(10), 1473-1482.

Zhang, Y., Luo, R., and Lytton, R. L. (2012). "Anisotropic Viscoelastic Properties of Undamaged Asphalt Mixtures”. J. Transp. Eng., 138(1), 75-89.

Zhang, Y., Luo, R., and Lytton, R. L. (2014). Anisotropic Characterization of Crack Growth in Tertiary Flow of Asphalt Mixtures in Compression. J Eng. Mechanics, 140(6), 04014032.

Gu, F., Luo, X., Zhang, Y., and Lytton, R. L. (2015a). "Using overlay test to evaluate fracture properties of field-aged asphalt concrete." Constr. Build. Mater., 101, 1059-1068.

Gu, F., Zhang, Y., Luo, X., Luo, R., and Lytton, R. L. (2015b). "Improved methodology to evaluate fracture properties of warm-mix asphalt using overlay test." Transp. Res. Record, 2506(1), 8-18.

Tan, S. A., Fwa, T. F., and Han, C. T. (2003). "Clogging evaluation of permeable bases." J. Transp. Eng., 129(3), 309-315.

Yong, C. F., McCarthy, D. T., and Deletic, A. (2013). "Predicting physical clogging of porous and permeable pavements." J. Hydrol., 481, 48-55.

Masad, E., Muhuthan, B., Shashidar, N., and Harman, T., "Internal structure characterization of asphalt concrete using image analysis." J. Comput. Civil Eng., 1999, 13-2, 88-95.

Al-Omari, A., and Masad, E. (2004). "Three dimensional simulation of fluid flow in Xray CT images of porous media.” Int. J. Numer. Anal. Meth. Geomech., 28, 13271360.

Hunter, A. E., and Airey, G. D. (2005). "Numerical modeling of asphalt mixture site permeability." Proceedings of the 84th Transportation Research Board Annual 
Meeting. (CD-ROM: Washington, DC).

Kutay, M. E., Aydilek, A. H., Masad E., and Harman, T. (2007). "Computational and experimental evaluation of hydraulic conductivity anisotropy in hot-mix asphalt." Int. J. Pavement Eng., 8(1), 29-43.

Masad, E., Al-Omari, A., and Chen, H. C. (2007). "Computations of permeability tensor coefficients and anisotropy of asphalt concrete based on microstructure simulation of fluid flow." Comp. Mater. Sci., 40, 449-459.

Gruber, I., Zinovik, I., Holzer, L., Flisch, A., and Poulikakos, L.D. (2012). “A computational study of the effect of structural anisotropy of porous asphalt on hydraulic conductivity." Constr. Build. Mater, 36, 66-77.

Chen, J., Li, H., Huang, X., and Wu, J. (2015). "Permeability loss of open graded friction course mixtures due to deformation-related and particle-related clogging: understanding from a laboratory investigation.” J. Mater. Civ. Eng., 27(11), 04015023.

Chen, J., Zhang, Y., Li, H., and Gao, Y. (2016). "Rutting-induced permeability loss of open graded friction course mixtures." J. Test. Eval., 44(2), 719-724.

Chen, J., Wang, H., and Zhu, H. (2017). "Investigation of permeability of open graded asphalt mixture considering effects of anisotropy and two-dimensional flow." Constr. Build. Mater., 145: 318-325. 